\title{
Evaluación de las Acciones Eólicas Transversales en Edificios de más de 5om mediante Métodos Analíticos
}

\author{
Evaluation of Cross-Wind actions in more than $50 \mathrm{~m}$ high buildings \\ by means of analytic methods
}

$\underline{\text { C. Muñoz }}^{(*)}$, I. Fortea $^{(*)}$, A. Albareda ${ }^{(*)}$

RESUMEN

En el presente trabajo se evalúa la relevancia que pueden llegar a adquirir las acciones eólicas transversales cuando se diseñan edificios aislados de más de 5om mediante las diferentes metodologías de análisis que establecen tres normas internacionales, el Eurocódigo, la norma australiana y la norma japonesa. El trabajo incluye tanto las acciones transversales que resultan de las oscilaciones periódicas del viento debido al desprendimiento de los vórtices, y que debiera ser considerado inevitablemente en cualquier edificio aislado, así como las acciones eólicas que son consecuencia del acoplamiento de frecuencias entre el propio desprendimiento de los vórtices y la frecuencia fundamental en sentido transversal del edificio, pudiendo dichas fuerzas llegar a condicionar el diseño de la estructura que debe hacer frente a las acciones horizontales del edificio.

Palabras clave: Edificios en altura, acción eólica transversal, acción dinámica, desprendimiento de vórtices, acoplamiento de frecuencias, resonancia, dinámica de fluidos.

\section{ABSTRACT}

This paper presents the relevance that can reach the cross-wind actions when isolated buildings with more that $50 \mathrm{~m}$ height are designed by means of the different analysis methodologies that are stablishes in those three international codes: Eurocode, Australian and Japanese. This paper includes both cross-wind loads that are the result of periodic wind oscillations due to vortex shedding and that should unavoidably be considered in any isolated building, and wind actions that are a consequence of the frequency coupling between the vortex shedding and the fundamental frequency of the building. These actions may determine the design of the structure that has to face the horizontal actions over the building .

Keywords: Tall buildings, cross-wind action, dynamic action, vortex-shedding, frequency coupling, resonance, fluid dynamics.

(*) Universidad Politécnica de Cataluña (España).

Persona de contacto/Corresponding author: carlos.munoz@upc.edu (C. Muñoz).

ORCID: https://orcid.org/oooo-0002-8996-1371 (C. Muñoz); https://orcid.org/oooo-0oo2-0016-6073 (I. Fortea); https://orcid.org/oooo-0002-2178-476X (A. Albareda)

Cómo citar este artículo/Citation: Muñoz, C.; Fortea, I.; Albareda, A. (2019). Evaluación de las Acciones Eólicas Transversales en Edificios de más de 5om mediante Métodos Analíticos. Informes de la Construcción, 71(554): e29o. https://doi.org/10.3989/ic.62723

Copyright: (C) 2019 CSIC. Este es un artículo de acceso abierto distribuido bajo los términos de la licencia de uso y distribución Creative Commons Reconocimiento 4.0 Internacional (CC BY 4.0). 


\section{INTRODUCCIÓN}

El estudio que aquí se presenta se centra en la evaluación de las acciones eólicas transversales que se producen en edificios aislados y flexibles, con alturas comprendidas entre los 50m y los 150m. En edificación dicho proceso acostumbra a basarse en métodos cuasi-estáticos que permiten evaluar la acción del viento, de naturaleza dinámica, como una acción estática, formada por la suma de la acción consecuencia de la velocidad media del viento, a la que se debe añadir la acción debida a las fluctuaciones y turbulencias del flujo. A partir de dichos métodos se determina la acción eólica longitudinal en aquellos edificios en los que los efectos dinámicos pueden ser despreciados. Del mismo modo, en diversos cuerpos normativos también se incluyen métodos de análisis para que el proyectista sea capaz de determinar, mediante métodos analíticos, las acciones dinámicas que se producen en las estructuras flexibles, donde los fenómenos aeroelásticos pueden ser relevantes.

Aunque es cierto que actualmente pueden existir diversos aspectos inciertos o que deben ser analizados de manera probabilística, como por ejemplo los parámetros climáticos a considerar en lugares concretos, la determinación relativa a la magnitud de las acciones longitudinales en los edificios rígidos o moderadamente flexibles puede ser realizada de manera inequívoca en aquellos casos en los que la geometría es simple, gracias a la aplicación de criterios prescriptivos. En el caso de edificios o entornos de geometría compleja es preciso recurrir a modelos numéricos basados en la dinámica computacional de fluidos o bien a túneles de viento experimentales que permitan analizar el comportamiento de flujos complejos de manera precisa.

Sin embargo, existen incertidumbres importantes a la hora de poder valorar cuantitativamente cual es la magnitud de las acciones eólicas transversales a la dirección del viento, aunque son ya muchos los estudios científicos que avalan la necesidad de que estas acciones transversales sean incluidas en el diseño de los edificios esbeltos, pudiendo tomar éstas incluso mayor relevancia que las acciones longitudinales, dependiendo de la altura y flexibilidad del edificio (1).

\section{BASE TEÓRICA}

En la determinación de la acción eólica transversal es importante diferenciar dos situaciones diferentes. En primer lugar, la acción eólica transversal que es consecuencia únicamente del comportamiento oscilatorio del flujo a través del edificio, y que acontece de manera inevitable para ciertos intervalos de velocidades del viento, independientemente de la rigidez y altura del edificio, pero que toma relevancia en las torres de planta cuadrada o circular. En segundo lugar, en determinadas situaciones se puede producir el acoplamiento entre el desprendimiento de los vórtices y la propia oscilación del edificio en sentido transversal al viento. En este segundo caso la acción eólica transversal adquiere una gran relevancia que llega a condicionar el diseño de la estructura.

El presente estudio analiza tres normas internacionales. Tomando como referencia el Eurocódigo 1991-1-4 (2), éste se compara con dos normas de reconocido prestigio, la norma australiana AS/NZS-1170.2:2011 (3) y las recomendaciones complementarias a la norma japonesa, AIJ, del año 2004 (4). Existen extensos estudios comparativos entre los métodos normativos y ensayos experimentales que confirman la precisión de dichos marcos normativos (5).

\subsection{El viento y el edificio como sistemas dinámicos}

Cuando se analiza la acción eólica es preciso tener en cuenta que el viento se debe considerar como un fenómeno aleatorio, donde algunos de los parámetros que lo definen únicamente se conocen de manera probabilística. Esta acción aleatoria, de carácter dinámico, habitualmente puede ser tratada de manera estática, gracias a la elevada frecuencia fundamental de los edificios si ésta se compara con la distribución de las fluctuaciones del viento en frecuencias (Figura 1). Destacan los espectros de Von-Karman y los espectros de Kaimal, teniendo

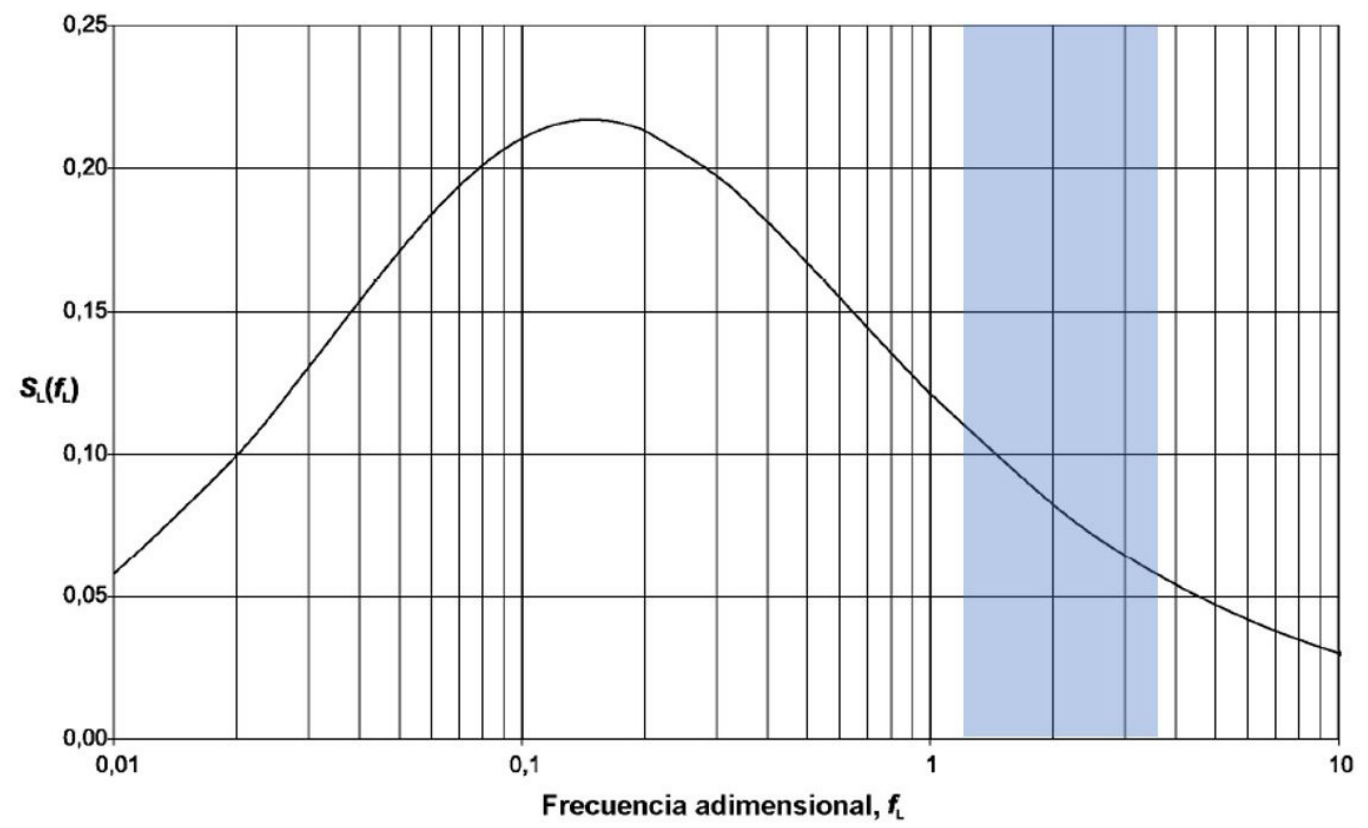

Figura 1. Función de densidad espectral de potencia $S_{\mathrm{I}}$. Imagen obtenida de Eurocódigo 1991-1-4, anejo B, Fig. B.1. Indicación en trama azul del ámbito en el que se encuentran los edificios en altura, entre 50m y 150m, y con frecuencias comprendidas entre $0,2 \mathrm{~Hz}$ y $1 \mathrm{~Hz}$. 
estos últimos una óptima aproximación a las descripciones empíricas de los espectros observados en la atmósfera (6).

Hay que destacar que la frecuencia $\mathrm{f}_{\mathrm{L}}$ es adimensional, siendo [1] la relación que existe entre la frecuencia del edificio, n, y dicha frecuencia adimensional, mediante la longitud turbulenta L(z) y la velocidad media del viento, $\mathrm{V}_{\mathrm{m}}$.

$$
\text { Frecuencia adimensional, } f_{L}(z, n)=\frac{n \cdot L(z)}{V_{m}(z)}
$$

Sin embargo, cuanto mayor sea la flexibilidad del edificio y, por lo tanto, menor sea su frecuencia fundamental, menos válida será la simplificación de considerar el viento como una acción estática. Las acciones del viento, en su componente dinámica, cada vez adoptaran una mayor relevancia en la determinación de las fuerzas que actúan sobre la estructura, hasta que llega un momento en el que, debido a la elevada flexibilidad del edificio, son los fenómenos aeroelásticos los que condicionarán el diseño del edificio.

Cuando el edificio se considera flexible, con frecuencias inferiores a 1,oHz, y se pueden producir desplazamientos y vibraciones en el edificio que provoquen que las fuerzas de inercia, dependientes de la masa y de la aceleración, y las fuerzas de amortiguamiento, tengan un valor similar a la propia acción del viento, ésta debe considerarse tal y como es en realidad, como una acción variable en el tiempo y en el espacio, debiendo en tal caso realizar un cálculo dinámico de la estructura.

Si se analiza una masa oscilante, sobre el que actúa una fuerza exterior dinámica como es el caso del viento, esta fuerza exterior debe estar en equilibrio con las fuerzas de inercia (producto de la masa por la aceleración), la fuerza de amortiguamiento y a la fuerza elástica, proporcional a la rigidez de la estructura y a su desplazamiento [2].

$$
f(t)=m \cdot \ddot{x}(t)+c \cdot \dot{x}(t)+k \cdot x(t)
$$

En la definición y resolución de la ecuación [2] existen dos aspectos que adquieren una elevada relevancia en el análisis y que pueden llegar a presentar una gran incertidumbre en el diseño del edificio, sobre todo en las fases iniciales del proyecto, la frecuencia del edificio y el amortiguamiento estructural. Respecto de la masa oscilante, existen estudios que permiten evaluar, de manera aproximada, la masa de una estructura desde las fases iniciales, conociendo únicamente la geometría del edificio (7).

La determinación de las frecuencias de los diferentes modos que definen el comportamiento dinámico de un edificio se puede realizar mediante un modelo de cálculo dinámico que considere los grados de libertad necesarios y que resuelva los autovectores de las matrices de $\mathrm{M}$ y K y de los autovalores del problema, o bien hacer uso, en las fases iniciales del proyecto, de diversas fórmulas simplificadas [3] (contenida en (2) y (8) y que permite una primera aproximación al problema, para edificios de más de 50m de altura) o ecuaciones que también permiten el análisis de las frecuencias para el análisis inicial de edificios en altura, como la contenida en (9) [4]. Se muestra en el gráfico 1 los valores que adopta la frecuencia para edificios comprendidos entre $50 \mathrm{Om}$ y $200 \mathrm{~m}$.

$$
\begin{gathered}
f_{n}=\frac{46}{h} \\
f_{n}=0,4 \cdot\left(\frac{100}{h}\right)^{1,6}
\end{gathered}
$$

Sin embargo, en muchas ocasiones el valor proporcionado por estas ecuaciones representa únicamente una primera aproximación a la solución final, dado que su precisión únicamente será adecuada en el caso de diseñar estructuras con una elevada rigidez frente a las acciones horizontales. En el caso de edificios flexibles se pueden producir diferencias en una proporción de hasta 2,5 a 1, siendo este hecho desfavorable en cuanto al análisis dinámico frente a las acciones eólicas.

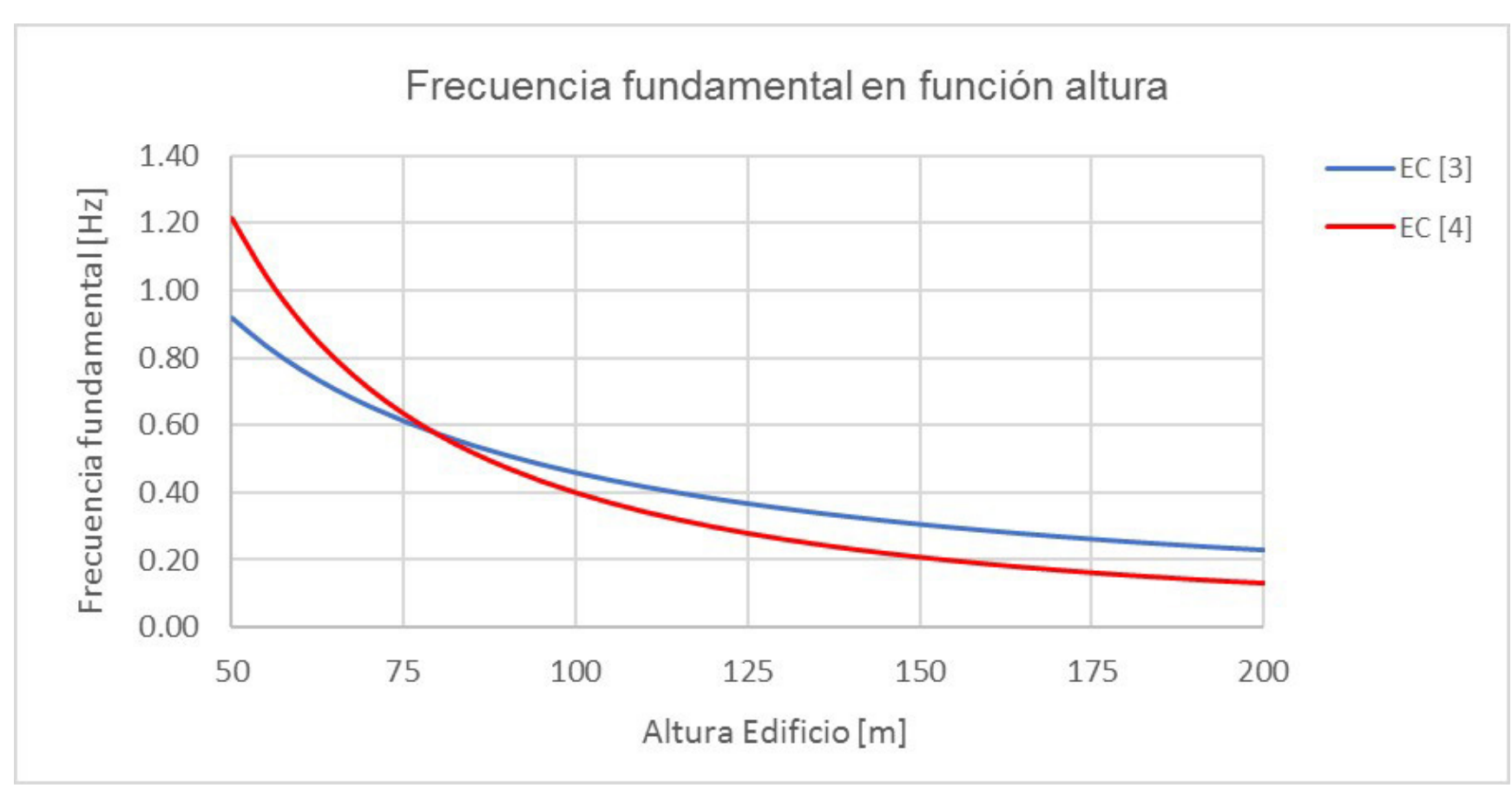

Gráfico 1. Frecuencia fundamental aproximada de las torres en función de su altura, según [3] y [4]. 
Respecto del amortiguamiento (entendido éste como la capacidad de disipación de energía cinética de una estructura vibrante en energía térmica), se considera únicamente el amortiguamiento interno debido a la componente estructural, siendo éste principalmente el que se produce debido al comportamiento del hormigón armado.

El amortiguamiento interno que genera el hormigón armado depende en gran medida del estado tensional al que se someta a la estructura. El amortiguamiento es máximo cuando la estructura se ha fisurado (distinguiendo una componente viscosa y una de rozamiento), pero se sigue manteniendo en unos niveles tensionales relativamente bajos. Sin embargo, cuando los esfuerzos se incrementan el amortiguamiento se va reduciendo paulatinamente, hasta alcanzarse, para estados elevados de tensión de las armaduras, unos valores de amortiguamiento incluso más reducidos que en los estados previos a la fisuración de la estructura (9). Se incluye en el apartado 2.3 los valores considerados en el presente estudio, en base a (8), (9), (10) y (11).

\subsection{Desprendimiento de vórtices}

Si se analiza el paso de un fluido a través de cualquier objeto sólido, el comportamiento de dicho fluido vendrá condicionado por la relación entre las fuerzas de inercia y las fuerzas viscosas, es decir, por lo que se conoce como Número de Reynolds [5].

$$
\frac{\text { Fuerzas de inercia }}{\text { Fuerzas viscosas }}=\frac{d \cdot U \cdot \rho}{\mu}
$$

Siendo d el tamaño de la sección transversal por la que pasa el fluido, U la velocidad del fluido, $\rho$ su densidad y $\mu$ su viscosidad dinámica. Dependiendo de la relación que adopten dichas fuerzas el comportamiento del flujo será laminar o tomará un carácter claramente turbulento. Dicho número de Reynolds también condiciona el paso del flujo a través del objeto, produciéndose, a medida que se incrementa la velocidad, un incremento en el comportamiento oscilatorio del flujo en la estela del objeto sólido.

Este comportamiento oscilatorio del flujo cuando el mismo atraviesa un objeto sólido en un régimen determinado de velocidad genera lo que se denominan las calles de Von Karman, un patrón repetitivo de vórtices rotacionales causados por la separación del flujo incidente en un objeto en función del tiempo (Figura 2). El desprendimiento alternado de los vórtices rotacionales a uno y otro lado del objeto sólido produce la oscilación del campo de presiones, que se traduce en

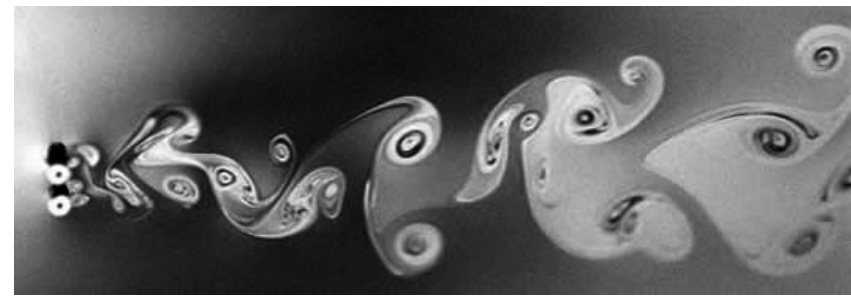

Figura 2. Calle de Von Karman compleja debido a la posición de dos cilindros paralelos. (Imagen extraída de la página web de la NASA y GES DISC).

la aplicación de una fuerza normal a la dirección del viento sobre el objeto, de naturaleza periódica (12) y (13).

Si la frecuencia con la que se desprenden los vórtices y, por lo tanto, la frecuencia con la que oscila la fuerza perpendicular al flujo, coincide o es similar con la frecuencia fundamental de la estructura se produce el acoplamiento de las frecuencias. La metodología de análisis determina una velocidad crítica del viento, a partir de la cual se puede producir el desprendimiento de vórtices en geometrías sencillas. Este análisis toma en consideración parámetros como la frecuencia fundamental de la estructura o la dimensión transversal de ésta. Según (2) no es preciso evaluar el desprendimiento de vórtices cuando la velocidad crítica [6] es un $25 \%$ superior a la velocidad media del viento.

$$
V_{\text {crit }}=\frac{b \cdot n_{1, y}}{S t}
$$

Siendo b el ancho del edificio normal a la dirección del flujo, $\mathrm{n}_{1, \mathrm{y}}$ es la frecuencia natural del modo de flexión considerado de la vibración perpendicular al viento, y St es el Número de Strouhal, de valor 0,12 para edificios de planta cuadrada. El ancho b se debe considerar allí donde la deformación modal es máxima. Del mismo modo, la frecuencia con la que los vórtices se desprenderán de un edificio de geometría sencilla se define de manera analítica según [7].

$$
f=\frac{S t \cdot V}{b}
$$

\subsection{Datos de partida}

La totalidad del estudio se extiende a cuatro esbelteces de edificios, todos ellos diseñados con un núcleo rígido central de hormigón armado para resistir las fuerzas horizontales. Para cada una de las cuatro alturas consideradas las propie-

\begin{tabular}{|c|c|c|c|c|c|}
\hline$\underset{[\mathrm{m}]}{\text { Altura }}-\mathbf{H}$ & $\begin{array}{c}\text { Dimensión en planta } \\
\text { BxD [m] }\end{array}$ & $\begin{array}{c}\text { Esbeltez según (2 y 3) } \\
{[\text { H/B }]}\end{array}$ & $\begin{array}{c}\text { Esbeltez según (4) } \\
{\left[H /\left(B^{*} D\right)^{o, 5}\right]}\end{array}$ & $\begin{array}{c}\text { Frecuencia } \\
\text { fundamental } \\
{[\mathrm{Hz}]}\end{array}$ & $\underset{[\%]}{\text { Amortiguamiento }}$ \\
\hline \multirow{2}{*}{$\begin{array}{c}50 \\
15 \text { plantas } \\
\end{array}$} & $25 \times 25$ & \multirow{2}{*}{2} & 2 & \multirow{2}{*}{0,92} & \multirow{2}{*}{2,5} \\
\hline & $25 \times 50$ & & 1,41 & & \\
\hline \multirow{2}{*}{$\begin{array}{c}75 \\
22 \text { plantas } \\
\end{array}$} & $25 \times 25$ & \multirow{2}{*}{3} & 3 & \multirow{2}{*}{0,61} & \multirow{2}{*}{2,0} \\
\hline & $25 \times 50$ & & 2,12 & & \\
\hline \multirow{2}{*}{$\begin{array}{c}100 \\
28 \text { plantas }\end{array}$} & $25 \times 25$ & \multirow{2}{*}{4} & 4 & \multirow{2}{*}{0,46} & \multirow{2}{*}{1,6} \\
\hline & $25 \times 50$ & & 2,83 & & \\
\hline \multirow{2}{*}{$\begin{array}{c}150 \\
42 \text { plantas }\end{array}$} & $25 \times 25$ & \multirow{2}{*}{6} & 6 & \multirow{2}{*}{0,31} & \multirow{2}{*}{1,6} \\
\hline & $25 \times 50$ & & 4,24 & & \\
\hline
\end{tabular}
dades incluidas quedan determinadas en la Tabla 1.

Tabla 1. Propiedades de los edificios considerados en el estudio. 
Se considera una velocidad básica del viento de $29 \mathrm{~m} / \mathrm{s}$ para un tiempo de medición de 10 minutos y un periodo de retorno de 50 años. La rugosidad del terreno considerada en todo el estudio es de tipo II, según (2).

Aunque actualmente los diferentes marcos normativos tienden a la estandarización de sus criterios, todavía existen diferencias fundamentales que se deben conocer y tener en cuenta. Algunas de estas diferencias son el tiempo de referencia para la medición de la velocidad básica del viento, que puede estar referida a 10 minutos o a 3 segundos, o la consideración de una curva de caracterización de la velocidad del viento en altura que puede ser de tipo logarítmica (Eurocódigo) o potencial (la norma japonesa, por ejemplo).

\section{SIMULACIONES / CÁLCULO}

El presente estudio toma como referencia la acción eólica longitudinal sobre el edificio según (2), calculando las fuerzas eólicas mediante la consideración de la presión correspondiente a la velocidad de pico, de los coeficientes de fuerza en el elemento estructural y del factor estructural $\mathrm{C}_{\mathrm{s}} \mathrm{C}_{\mathrm{d}}$, dependiente este último del factor de respuesta de fondo y del factor de respuesta debido a la resonancia (14).

\subsection{Acción eólica longitudinal}

Tomando como referencia la acción eólica longitudinal se puede valorar la influencia que puede llegar a tomar en cada caso la acción eólica transversal, comparada ésta última como porcentaje de la acción eólica longitudinal.

A partir de la aplicación de la metodología de análisis incluida en (2) se muestra en la tabla 2 los factores de respuesta y el coeficiente estructural que afecta a los edificios analizados, así como la fuerza global que se produce en cada caso en sentido paralelo a la dirección del viento.

Asumiendo que el factor de respuesta de fondo es igual a la unidad y, por lo tanto, también el factor de tamaño $\mathrm{C}_{\mathrm{s}}$, y comparando en este caso los valores del factor dinámico $\mathrm{C}_{\mathrm{d}}$ con los obtenidos en (3), se pueden apreciar grandes similitudes entre los factores dinámicos determinados en (2) y en (3) (Tabla 2).
Se observa una gran similitud entre los valores obtenidos en (2) con los obtenidos en (4), con diferencias en la acción longitudinal del viento que no dista más de un $10 \%$ respecto del Eurocódigo (ver tabla 3), a pesar de los diferentes planteamientos en sus metodologías de análisis.

Tabla 3. Comparación de la fuerza eólica longitudinal definida según Eurocódigo y AIJ para edificios de esbeltez de 3, 4 y 6.

\begin{tabular}{|c|c|c|c|c|}
\hline \multirow{2}{*}{ BxD } & \multirow{2}{*}{ Norma } & \multicolumn{3}{|c|}{$\begin{array}{l}\text { Fuerza longitudinal } \\
{[\mathrm{kN}]}\end{array}$} \\
\hline & & $\mathrm{H}: 75 \mathrm{~m}$ & H: 100m & H: $150 m$ \\
\hline \multirow{3}{*}{$25 \times 25 \mathrm{~m}$} & Eurocódigo & 4.162 & 6.088 & 10.353 \\
\hline & AIJ & 3.852 & 5.545 & 9.514 \\
\hline & Diferencia [\%] & 7,4 & 8,9 & 8,1 \\
\hline \multirow{3}{*}{$25 \times 50 m$} & Eurocódigo & 3.246 & 4.784 & 8.135 \\
\hline & AIJ & 3.363 & 4.850 & 8.267 \\
\hline & Diferencia [\%] & 3,5 & 1,4 & 1,6 \\
\hline
\end{tabular}

Se verifica que las diferencias porcentuales entre las tres normas incluidas en el estudio son siempre inferiores al $10 \%$. Esto permite analizar las acciones eólicas transversales de los tres cuerpos normativos de manera coherente.

\subsection{Acción eólica transversal}

Se resumen a continuación las diferentes metodologías de análisis incluidas en las tres normas de referencia para la determinación de las acciones eólicas transversales en los edificios.

\subsubsection{Metodología de análisis del Eurocódigo 1991-1-4}

El Eurocódigo no incluye de manera explícita una metodología de análisis para evaluar las acciones eólicas transversales en aquellos edificios moderadamente flexibles en los que no se produce el acoplamiento por desprendimiento de vórtices, pero sí que deben ser tenidos en cuenta los efectos dinámicos del edificio en conjunción con el movimiento oscilatorio del flujo eólico. La propia norma, en su apartado 3.5, determina que el marco de la misma queda limitado a la respuesta dinámica a las turbulencias en la dirección del viento en resonancia con las vibraciones en la dirección del viento de un modo fundamental de flexión de signo constante. Únicamente, en el apartado 7.1.2, determina la necesidad de "tener en cuenta

Tabla 2. Factores de respuesta y factores dinámicos para edificios de esbeltez 2, 3, 4 y 6, según (2) y (3). Fuerza eólica global longitudinal en la base.

\begin{tabular}{|c|c|c|c|c|c|c|c|c|c|c|}
\hline \multirow{2}{*}{$\underset{[\mathbf{m}]}{\mathbf{H}}$} & \multirow[b]{2}{*}{ Planta BxD } & \multicolumn{5}{|c|}{ Eurocódigo } & \multicolumn{4}{|c|}{ AS/NZS } \\
\hline & & $\mathbf{B}^{2}$ & $\mathbf{R}^{2}$ & $\mathbf{C}_{\mathrm{s}}$ & $\mathbf{C}_{\mathrm{d}}$ & $\begin{array}{c}\text { Fuerza longitudinal } \\
{[\mathrm{kN}]}\end{array}$ & $\begin{array}{c}\mathbf{B}_{\mathrm{s}} \\
\mathbf{S}=\mathbf{o m}\end{array}$ & $\underset{S=O \mathbf{m}}{\mathbf{C}_{\mathrm{dyn}}}$ & $\underset{\mathbf{S}}{\mathbf{B}=\mathbf{h}}$ & $\begin{array}{c}C_{\text {dyn }} \\
\mathbf{S}=\mathbf{h}\end{array}$ \\
\hline \multirow{3}{*}{50} & $25 \times 25$ & \multirow{2}{*}{0,59} & \multirow{2}{*}{0,09} & \multirow{2}{*}{0,88} & \multirow{2}{*}{1,02} & 2.529 & \multirow{2}{*}{0,81} & \multirow{2}{*}{0,97} & \multirow{2}{*}{0,88} & \multirow{2}{*}{1,01} \\
\hline & $25 \times 50$ & & & & & 1.987 & & & & \\
\hline & $50 \times 25$ & 0,54 & 0,05 & 0,87 & 1,00 & 5.404 & 0,75 & 0,94 & 0,79 & 0,97 \\
\hline \multirow{3}{*}{75} & $25 \times 25$ & \multirow{2}{*}{0,59} & \multirow{2}{*}{0,19} & \multirow{2}{*}{0,88} & \multirow{2}{*}{1,05} & 4.162 & \multirow{2}{*}{0,77} & \multirow{2}{*}{0,98} & \multirow{2}{*}{0,89} & \multirow{2}{*}{1,05} \\
\hline & $25 \times 50$ & & & & & 3.246 & & & & \\
\hline & $50 \times 25$ & 0,55 & 0,11 & 0,87 & 1,02 & 8.786 & 0,73 & 0,96 & 0,81 & 1,00 \\
\hline \multirow{3}{*}{100} & $25 \times 25$ & \multirow{2}{*}{0,57} & \multirow{2}{*}{0,34} & \multirow{2}{*}{0,88} & \multirow{2}{*}{1,10} & 6.088 & \multirow{2}{*}{0,74} & \multirow{2}{*}{0,97} & \multirow{2}{*}{0,90} & \multirow{2}{*}{1,05} \\
\hline & $25 \times 50$ & & & & & 4.784 & & & & \\
\hline & $50 \times 25$ & 0,54 & 0,21 & 0,87 & 1,05 & 12.827 & 0,71 & 0,97 & 0,82 & 1,04 \\
\hline \multirow{3}{*}{150} & $25 \times 25$ & \multirow{2}{*}{0,55} & \multirow{2}{*}{0,52} & \multirow{2}{*}{0,87} & \multirow{2}{*}{1,14} & 10.353 & \multirow{2}{*}{0,68} & 102 & 0.01 & 116 \\
\hline & $25 \times 50$ & & & & & 8.135 & & 1,03 & , & 1,10 \\
\hline & $50 \times 25$ & 0,53 & 0,36 & 0,86 & 1,09 & 21.009 & 0,68 & 0,99 & 0,83 & 1,10 \\
\hline
\end{tabular}


los efectos de fluctuaciones instantáneas del viento que produzcan cargas asimétricas y no compensadas en aquellas estructuras sensibles a este tipo de acciones”.

Con el objetivo de poder determinar un valor de acción eólica transversal en aquellos edificios menos flexibles, los autores del presente estudio toman como referencia los coeficientes eólicos de succión que define el Eurocódigo en su apartado 7.2. El presente estudio define la acción eólica transversal, según (2), como el efecto de la succión en una única fachada lateral, aunque esto no esté explícitamente indicado.

Sin embargo, en aquellos edificios más esbeltos y flexibles, susceptibles de padecer fenómenos aeroelásticos, como es el acoplamiento de frecuencias por el desprendimiento de vórtices, el Eurocódigo incluye un método que permite evaluar el efecto de las vibraciones inducidas por dicho desprendimiento de los vórtices a partir del efecto de la fuerza de inercia por unidad de longitud $F_{w}(s)$ [8], actuando perpendicularmente a la dirección del viento.

$$
F_{w}(s)=m(s) \cdot\left(2 \cdot \pi \cdot n_{i, y}\right)^{2} \cdot \Phi_{1, y}(s) \cdot Y_{F, \max }
$$

Siendo $m(s)$ la masa vibrante del edificio por unidad de longitud, $n_{i, y}$ la frecuencia natural de la estructura en el sentido transversal a la dirección eólica, $\phi_{1, y}(s)$ la forma modal de la estructura normalizada a 1 en el punto de máximo desplazamiento y $Y_{F, \max }$ es el valor del máximo desplazamiento a lo largo del tiempo del punto en el que $\phi_{1, y}(s)$ tiene valor máximo.

Para evaluar $Y_{F, \max }$, es decir, las amplitudes generadas en sentido transversal a la acción eólica, como consecuencia del desprendimiento de los vórtices, el Eurocódigo incluye dos métodos diferentes, el modelo basado en la resonancia de vórtices [9] (15) y el modelo espectral [10]. Ambos métodos difieren en la consideración de la componente turbulenta del viento, y mientras que el primero es válido en aquellos climas con vientos sometidos a fluctuaciones turbulentas importantes (España, por ejemplo), el segundo es de aplicación en aquellas regiones climáticas muy frías y estratificadas, por ejemplo, la costa del Norte de Europa.

$$
\frac{Y_{F, \max }}{b}=\frac{1}{S t^{2}} \cdot \frac{1}{S c} \cdot K \cdot K_{w} \cdot c_{l a t}
$$

Siendo St el número de Strouhal, Sc el número de Scruton, K el factor de forma modal, $\mathrm{K}_{\mathrm{w}}$ el factor de longitud efectiva de correlación, que sirve para incluir las fuerzas aeroelásticas, y $\mathrm{c}_{\text {lat }}$ el coeficiente de fuerza lateral.

$$
Y_{F, \max }=\sigma_{y} \cdot k_{p}
$$

Siendo $\sigma_{y}$ la desviación típica del desplazamiento y $k_{p}$ el factor de pico.

Todo el trabajo que aquí se presenta según Eurocódigo se realiza mediante la consideración del modelo basado en la resonancia de vórtices.

\subsubsection{Metodología de análisis de la norma australiana}

La norma australiana recoge métodos para la determinación de las fuerzas estáticas y los momentos de vuelco en la base para edificios en altura, con frecuencias fundamentales infe- riores a $1 \mathrm{~Hz}$. El método, de aplicación mediante la incorporación en el cálculo de un factor dinámico de respuesta, se limita a estructuras con frecuencias fundamentales no menores que $0,2 \mathrm{~Hz}$ y edificios con alturas inferiores a los 200m, y en aquellas estructuras donde los dos primeros modos fundamentales de oscilación no pueden acoplarse.

El método cubre un amplio rango de frecuencias, identificando tanto el correspondiente a la entrada en resonancia del edificio como a las oscilaciones de la carga transversal debido al desprendimiento de vórtices fuera del rango de acoplamiento. Se calcula la fuerza por unidad de longitud que aplica al edificio según la expresión [11], contenida en la sección 6 de (3).

$$
w_{e q}=\frac{1}{2} \cdot \rho_{\text {aire }} \cdot\left(V_{\text {des }, \theta}\right)^{2} \cdot d \cdot C_{f i g} C_{d y n}
$$

Evaluando la velocidad del viento en la parte superior del edificio, y siendo d la dimensión de la torre paralela a la dirección eólica. El método del Factor de respuesta Dinámica, para la determinación de la acción transversal, se basa en la definición del factor $C_{f i g} D_{d u n}$ que incluye el factor de forma y el factor de amplificación dinámica en un único parámetro, definido según [12].

$$
C_{f i g} C_{d y n}=1,5 \cdot g_{R} \cdot\left(\frac{b}{d}\right) \cdot \frac{k_{m}}{\left(1+g_{v} \cdot I_{h}\right)^{2}} \cdot\left(\frac{z}{h}\right)^{k} \cdot \sqrt{\frac{\pi \cdot C_{f s}}{\xi}}
$$

Siendo $g_{R}$ el factor de pico para la respuesta de resonancia, b y d las dimensiones en planta de la torre, $k_{m}$ el factor corrector de la forma modal para las aceleraciones transversales a la dirección del viento, $g_{v}$ el factor de pico para las fluctuaciones corriente arriba del flujo, de valor constante $3,7, I_{h}$ la intensidad turbulenta en la parte superior del edificio, z la altura en la que se evalúa la acción eólica, $h$ la altura del edificio, $C_{f_{s}}$ el coeficiente espectral de la fuerza transversal, evaluado a partir de la velocidad reducida del viento, y $\xi$ el ratio de amortiguamiento estructural respecto del amortiguamiento crítico.

\subsubsection{Metodología de análisis de la norma japonesa}

La norma japonesa diferencia tres situaciones diferentes, en función de la esbeltez y rigidez del edificio. Para edificios poco esbeltos, con valores inferiores a 3 , incluye la acción eólica transversal de manera combinatoria, representando la acción transversal un porcentaje de la acción longitudinal, según la proporción en planta del edificio (16). Cuando la esbeltez del edificio es igual o mayor que 3 , entonces se deben determinar las acciones transversales de manera analítica [13].

$$
W_{L}=3 \cdot q_{H} \cdot C_{L}^{\prime} \cdot A \cdot \frac{Z}{H} \cdot g_{L} \cdot \sqrt{\left(1+\varnothing_{L}^{2} \cdot R_{L}\right.}
$$

Siendo $q_{H}$ la presión correspondiente a la velocidad de cálculo en la parte alta del edificio, $g_{L}$ el factor de pico, $\varnothing_{L}$ el factor corrector para la forma modal, $R_{L}$ el factor de resonancia y C' un factor que depende de la proporción entre las dimensiones de la planta B y $\mathrm{D}$.

Si se cumplen las inecuaciones [14] y [15] la norma japonesa determina que es necesaria la verificación del desprendimiento de vórtices, incluyendo para ello únicamente un método analítico aplicable en elementos de sección circular. 


$$
\begin{gathered}
\frac{H}{\sqrt{D \cdot B}} \geq 4 \\
\frac{U_{H}}{f_{L} \cdot \sqrt{B \cdot D}} \geq 0,83 \cdot U_{L C R}^{*}
\end{gathered}
$$

Siendo $\mathrm{H}$ la altura del edificio, B y D las dimensiones en planta, $U_{H}$ la velocidad del viento en la parte alta del edificio, $f_{L}$ la frecuencia en dirección transversal al viento y $U_{L c R}$ la velocidad inicial adimensional.

\section{RESULTADOS Y DISCUSIÓN}

En el estudio de las fuerzas eólicas transversales se deben diferenciar dos situaciones completamente diferentes a la hora de analizar los resultados obtenidos. Se ha visto como el Eurocódigo no incluye de manera explícita un método que defina la carga eólica transversal, pero sí que determina los coeficientes de succión que deben considerarse en cada una de las fachadas laterales. Es por ese motivo que en la definición de las acciones según (2) en el presente estudio se determina la fuerza eólica transversal en edificios rígidos mediante la consideración de dichos coeficientes de succión.

Además, existe un umbral a partir del cual es preciso evaluar de manera detallada la posibilidad de que se produzca el acoplamiento entre el desprendimiento de vórtices y el modo fundamental transversal del edificio, siendo entonces fundamental la consideración de las acciones eólicas transversales en el diseño de la estructura.

\subsection{Valores numéricos obtenidos del análisis}

Si se comparan los resultados obtenidos en las tres normas analizadas (Tabla 4), para las frecuencias determinadas según la ecuación [3] en 4 alturas diferentes de edificios, se puede observar que considerar los coeficientes eólicos de succión incluidos en el Eurocódigo en una única fachada lateral permite eva- luar la fluctuación del flujo eólico en edificios de hasta 100m de altura y frecuencias fundamentales superiores a $0,5 \mathrm{~Hz}$ con un grado aceptable de aproximación para los casos analizados.

Sin embargo, cuando se analiza la acción eólica transversal en un edificio de $150 \mathrm{~m}$ y frecuencia fundamental de $0,31 \mathrm{~Hz}$ la acción eólica que se determina mediante el Eurocódigo, sin incluir la flexibilidad del propio edificio, queda claramente infravalorada respecto de los valores que se determinan mediante la aplicación de la norma australiana y de la japonesa, existiendo incrementos próximos al 78\% si se compara con la AIJ. Los valores obtenidos mediante estas dos últimas normas de referencia son sensiblemente similares.

La norma australiana, por ejemplo, permite analizar en detalle la acción eólica transversal que se produce en edificios flexibles en función de la altura. Se ha visto que en edificios con esbeltez de 3 y frecuencias superiores a $0,5 \mathrm{~Hz}$ los valores de empuje lateral son similares a los determinados en el Eurocódigo si se aplican los coeficientes laterales de succión en una única fachada. Sin embargo, si se analiza de manera detallada la acción transversal en edificios flexibles con esbelteces de 6, se puede apreciar como la presión lateral puede alcanzar en la parte alta de la torre valores próximos a $5 \mathrm{kN} / \mathrm{m}^{2}$ (Tabla 5 ).

Es importante destacar que estos valores de fuerza transversal se producen como consecuencia de la oscilación del flujo en el edificio, sin alcanzarse el acoplamiento entre el desprendimiento de los vórtices y la frecuencia fundamental de la estructura. Se puede observar, para las casuísticas analizadas, que en edificios de esbeltez de 3 la fuerza transversal representa aproximadamente un 65\% de la acción eólica longitudinal. Sin embargo, a medida que la esbeltez y la flexibilidad se incrementan el porcentaje también se ve incrementado. Por ejemplo, para el edificio de esbeltez de 6, la acción eólica transversal llega a representar un 108\% de la acción longitudinal, valores producidos sin llegar a alcanzarse el acoplamiento de frecuencias en ningún caso. En el gráfico 2 se

Tabla 4. Estudio comparativo de la acción eólica transversal en la base de 4 esbelteces de edificio, todos ellos de planta cuadrada de $25 \mathrm{~m}$ de lado, en función de las tres normas incluidas en el presente estudio.

\begin{tabular}{|c|c|c|c|c|c|}
\hline \multirow{2}{*}{$\begin{array}{c}\text { Norma de } \\
\text { referencia }\end{array}$} & \multirow{2}{*}{ Método } & \multicolumn{4}{|c|}{ Fuerza transversal [kN] $\mathbf{f}_{\mathbf{n}}=\mathbf{4 6} / \mathbf{H}$} \\
\cline { 3 - 6 } & H: 50m & H: $\mathbf{7 5 m}$ & H: 100m & H: 150m \\
\hline \multirow{2}{*}{ Eurocódigo } & Presión fachada lateral & 1.855 & 2.954 & 4.207 & 6.507 \\
\cline { 2 - 6 } & Desprendimiento vórtices & \multicolumn{2}{|c|}{ Verificar sólo si $\mathrm{f}_{\mathrm{n}}<0,22 \mathrm{~Hz}$} & Verificar sólo si $\mathrm{f}_{\mathrm{n}}<0,24 \mathrm{~Hz}$ \\
\hline \multirow{2}{*}{ AS/NZS } & $\begin{array}{c}\text { Método del factor de } \\
\text { amplificación dinámica }\end{array}$ & Fuera rango & 2.669 & 4.100 & 10.181 \\
\hline AIJ & Evaluación analítica & 1.542 & 2.796 & 4.409 & 11.598 \\
\hline
\end{tabular}

\begin{tabular}{|c|c|c|c|c|}
\hline \multirow[b]{3}{*}{ Altura analizada } & \multicolumn{4}{|c|}{ AS/NZS Presión eólica transversal $\left[\mathrm{kN} / \mathrm{m}^{2}\right]$} \\
\hline & \multirow{2}{*}{$\begin{array}{c}\text { Edificio } 75 \mathrm{~m} \\
(3: 1: 1)\end{array}$} & \multicolumn{3}{|c|}{ Edificio 150m [h:b:d] } \\
\hline & & $(6: 1: 1)$ & $(6: 2: 1)$ & $(6: 1: 2)$ \\
\hline 25 & 0,71 & 0,78 & 0,83 & 0,34 \\
\hline 50 & 1,42 & 1,54 & 1,65 & 0,67 \\
\hline 75 & 2,14 & 2,32 & 2,50 & 1,01 \\
\hline 100 & & 3,11 & 3,33 & 1,34 \\
\hline 125 & & 3,89 & 4,16 & 1,68 \\
\hline 150 & & 4,65 & 4,98 & 2,03 \\
\hline Cortante transversal $[\mathrm{kN}]$ & 2.669 & 10.181 & 10.908 & 8.826 \\
\hline $\begin{array}{l}\text { Porcentaje respecto de acción eólica } \\
\text { longitudinal (EC) }\end{array}$ & $65 \%$ & $98 \%$ & $50 \%$ & $108 \%$ \\
\hline
\end{tabular}

Tabla 5. Presión eólica transversal en función de la altura, en edificios de esbeltez 3 y 6 , evaluada en base a (3). 


\section{Fuerza total consecuencia del desprendimiento de vórtices BxD: $25 \times 25 \mathrm{~m}$}

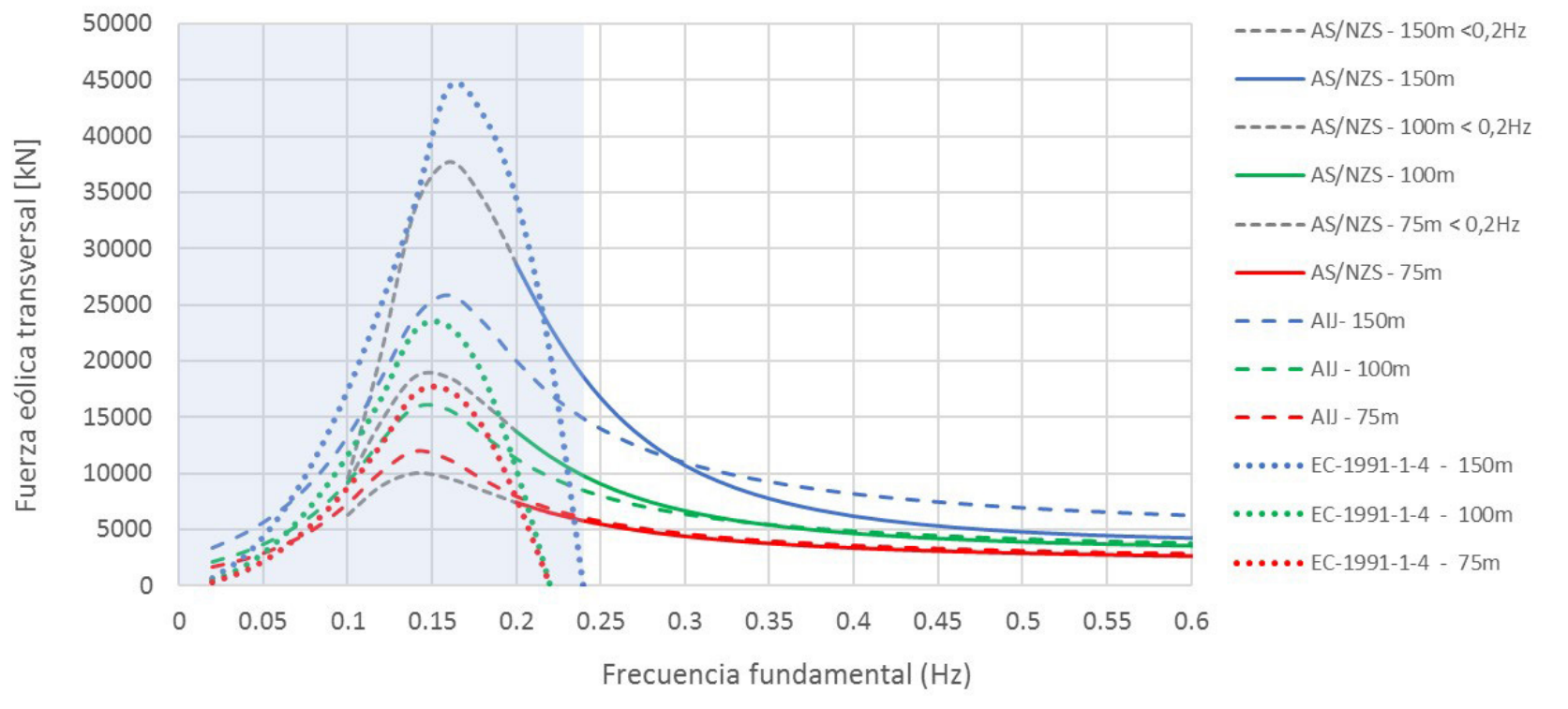

Gráfico 2. Fuerza eólica transversal en la base de edificios de esbeltez 3, 4 y 6 y planta de 25x25m, según las tres normas analizadas, en función de la frecuencia. Intervalo fuera de rango para la norma australiana por debajo de $0,2 \mathrm{~Hz}$, en línea gris discontinua.

extiende el análisis a un intervalo más amplio de frecuencias fundamentales en el modo transversal para tres esbelteces de edificios $(3,4$ y 6$)$.

Se observa, para todas las esbelteces analizadas, un pico máximo de fuerza transversal para una frecuencia de $0,15^{-}$ $0,17 \mathrm{~Hz}$, correspondiendo ésta al valor en el que se produce el máximo acoplamiento entre el desprendimiento del flujo y la propia estructura (resonancia de la estructura). En el gráfico se puede ver tramado en color azul el ámbito en el que, según el Eurocódigo, es necesario evaluar el posible acoplamiento entre el desprendimiento de vórtices y la propia estructura, es decir, para frecuencias inferiores a $0,24 \mathrm{~Hz}$ en el caso de un edificio de $150 \mathrm{~m}$ de altura y $0,22 \mathrm{~Hz}$ para los edificios de 75 y $100 m$ de altura. Por ejemplo, para el edificio de $150 \mathrm{~m}$ de altura la fuerza transversal en el momento de producirse el acoplamiento de frecuencias es del orden de cuatro veces y media superior a la acción que se produciría para frecuencias fundamentales de $0,3 \mathrm{~Hz}$.

Uno de los aspectos más relevantes es que existe un intervalo de frecuencias para el que el Eurocódigo no determina ninguna acción transversal relevante, pero que sin embargo si que es fundamental analizar dichas acciones, ya que son incrementadas debido a la propia flexibilidad del edificio. $\mathrm{Si}$ se analiza el gráfico 2 se observa como existe un intervalo de frecuencias, comprendido aproximadamente entre $0,24 \mathrm{~Hz}$ y $0,5 \mathrm{~Hz}$ para los edificios de mayor esbeltez y entre $0,22 \mathrm{~Hz}$ y $0,3 \mathrm{~Hz}$ para los de esbeltez de 3 y 4 en los que se aprecia un incremento muy significativo en las acciones transversales.

Se puede realizar un análisis similar, mediante la norma australiana, evaluando cual es la respuesta de un edificio de $150 \mathrm{~m}$ de altura y 3 frecuencias fundamentales diferentes, dependiendo de la velocidad del viento en la parte superior de la torre (gráfico 3). Si se analiza la fuerza eólica transversal total en la base del edificio para la velocidad máxima de pico que puede producirse en España, para un periodo de retorno de 100 años, se observa que la diferencia entre una frecuen- cia fundamental de $0,2 \mathrm{~Hz}$ y de $0,3 \mathrm{~Hz}$ está en una relación de 2,5:1, reduciéndose la fuerza eólica transversal de 40.00okN a $16.000 \mathrm{kN}$ aproximadamente. Si bien es cierto que un valor de frecuencia de $0,3 \mathrm{~Hz}$ impide en este caso el acoplamiento por desprendimiento de vórtices, un valor de $0,24 \mathrm{~Hz}$ representa ya el umbral en el que sería preciso analizarlo, siendo $0,20 \mathrm{~Hz}$ un valor muy próximo al valor pico de acoplamiento de frecuencias.

\subsection{Comparación mediante la Dinámica Computacional de Fluidos}

Recurriendo a simulaciones basadas en la dinámica computacional de fluidos se analiza cual es la oscilación transversal del flujo para diferentes velocidades medias del viento. Tomando como referencia un edificio de planta cuadrada de 25x25m se analiza la oscilación para una velocidad media de $40 \mathrm{~m} / \mathrm{s}$, equivalente a una velocidad básica de $29 \mathrm{~m} / \mathrm{s}$ en un terreno de rugosidad II, a $75 \mathrm{~m}$ de altura aproximadamente. Estos valores se comparan con una velocidad de 30m/s (periodo de retorno de 1 año) y 10m/s.

Tanto la simulación correspondiente a la máxima velocidad del flujo (40m/s) como para un periodo de retorno de 1 año identifican inequívocamente la oscilación del flujo, con coeficientes globales máximos y mínimos de fuerza similares para ambas simulaciones del orden de 2,5, con un valor medio nulo (gráfico 4). Estos valores pico, cuyo comportamiento se asimila a una onda sinusoidal, representa los valores máximos de succión en los dos sentidos de la oscilación, positiva y negativa. En este punto es importante indicar que los valores próximos a 2,5 se reducirían a 1,0 si seleccionásemos duraciones de la ráfaga de 3 s, que son los que pueden afectar a las estructuras de edificación. Estas magnitudes son coincidentes, por ejemplo, con los coeficientes de presión que define el Eurocódigo para las fachadas laterales en los edificios.

Aplicando la Transformada Rápida de Fourier (FFT) (gráfico 4) para un flujo a 40m/s se obtiene aquel valor con mayor 


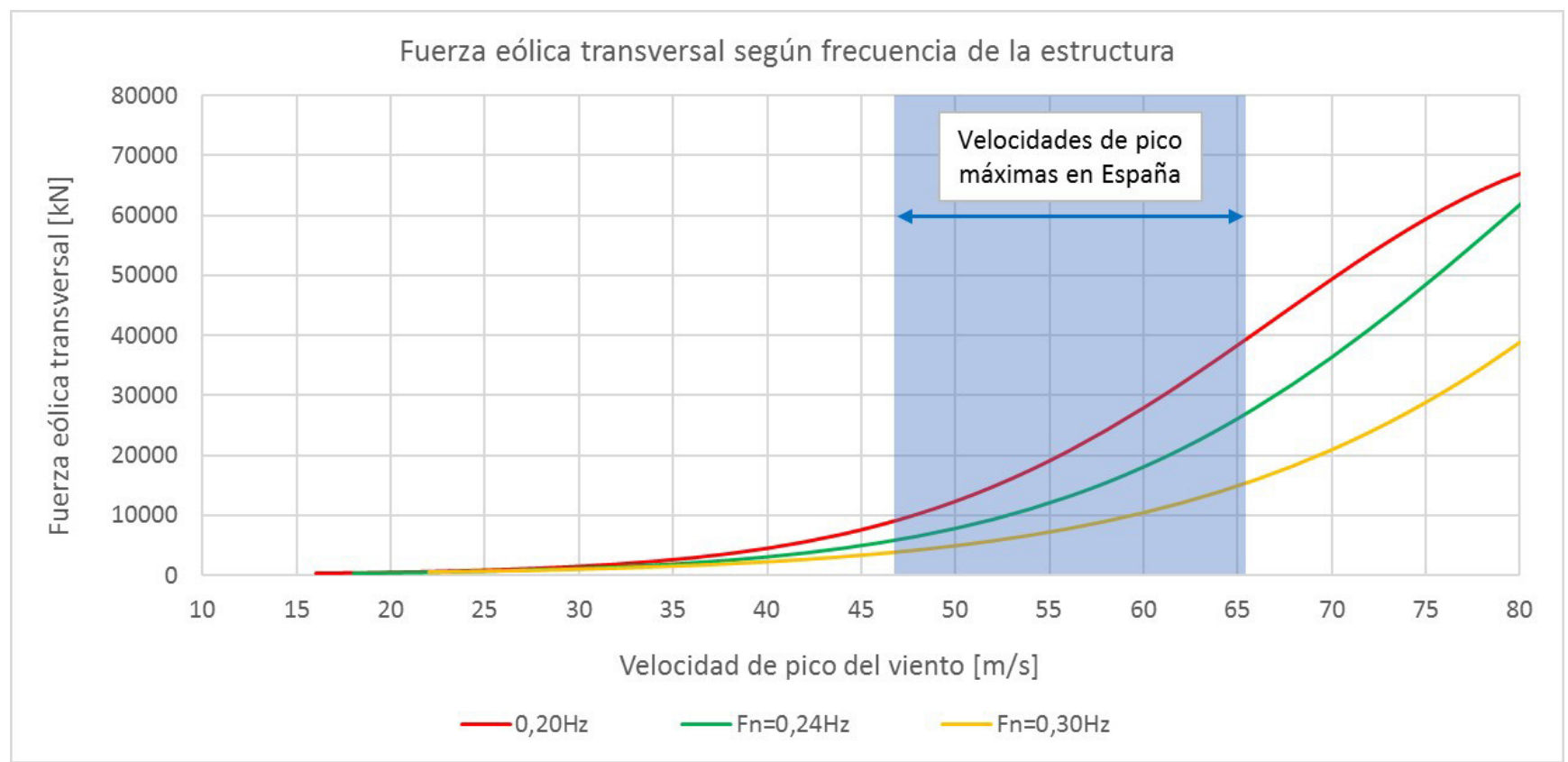

Gráfico 3. Fuerza eólica transversal según método incluido en (3), en la base de edificios de 15om de altura y esbeltez de 6, en función de la velocidad máxima de pico del viento.
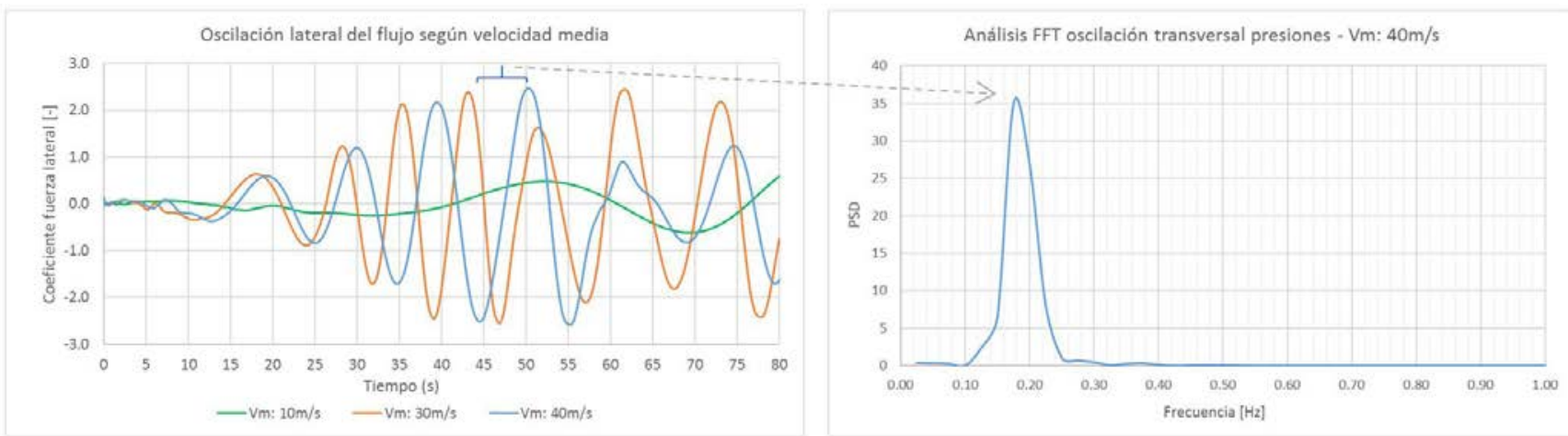

Gráfico 4. Oscilación del coeficiente de fuerza lateral para tres velocidades medias del viento. Evaluación mediante Dinámica Computacional de Fluidos. Análisis espectral de frecuencias mediante Transformada Rápida de Fourier (FFT) de la oscilación lateral del coeficiente de fuerza lateral en edificios de $25 \times 25 \mathrm{~m}$, para una velocidad media de $40 \mathrm{~m} / \mathrm{s}$.

densidad espectral de frecuencias (17). Es preciso tener en cuenta que, si se aplica la FFT a la señal obtenida, al ser el periodo una oscilación completa, se obtendría la frecuencia mitad de la que realmente corresponde al desprendimiento de los vórtices, en este caso para 0,09Hz. Es preciso aplicar la FFT teniendo en cuenta esta consideración, de manera que la frecuencia obtenida corresponda al desprendimiento de los vórtices, es decir, $0,18 \mathrm{~Hz}$ en la simulación. Si se aplica la Ecuación [7], analizando cual es la frecuencia de desprendimiento de vórtices, se obtiene un valor de $0,19 \mathrm{~Hz}$, valor que coincide con los estudios comparativos llevados a cabo mediante dinámica computacional de fluidos, con un margen de error próximo al $5 \%$.

Si se observa el comportamiento del flujo, tomando como referencia la simulación con la velocidad de $40 \mathrm{~m} / \mathrm{s}$, para un instante de tiempo en el que el coeficiente de fuerza lateral es máximo $(\mathrm{t}=5 \mathrm{Os})$, se puede observar el comportamiento oscilatorio del campo de presiones (Figura 3), con valores de succión máximo en una única cara del edificio, de valor de pico de 2.800 Pa.
Estas simulaciones no tienen en cuenta el efecto de acoplamiento de frecuencias debido a la flexibilidad del edificio. Esto evidencia la necesidad de considerar las fuerzas transversales en toda construcción de proporción cuadrada y aislada, independientemente de la flexibilidad que tenga la construcción y la propia necesidad de incluir el efecto del acoplamiento de frecuencias.

\section{CONCLUSIONES}

En el presente trabajo se ha evaluado de manera cuantitativa cual es la incidencia que puede llegar a alcanzar las acciones eólicas transversales en el diseño de edificios. Se ha podido verificar la necesidad de incluir las acciones eólicas transversales en el diseño de edificios, habiendo estudiado detalladamente aquellos de planta cuadrada y de proporción 1:2. Mientras que para aquellos edificios de menos de 100m y con frecuencias superiores a $0,5 \mathrm{~Hz}$ aproximadamente, la consideración de la fuerza eólica transversal mediante la incorporación de un valor de succión en una única fachada lateral, aplicando los coeficiente del Eurocódigo de manera asimétri- 


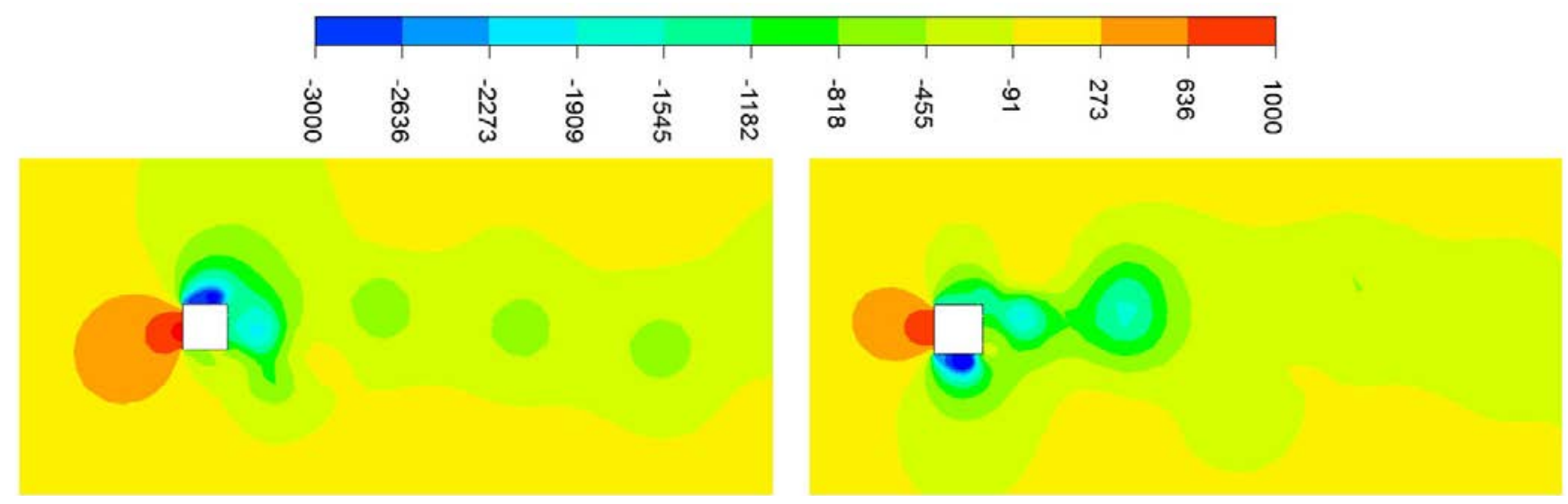

Figura 3. Campo de presiones oscilatorias del flujo [Pa] en la estela de un edificio de planta cuadrada analizado mediante Dinámica Computacional de Fluidos, para un tiempo $t=50$ y un incremento de mitad del periodo, $\mathrm{T} / 2$. Flujo eólico en la imagen de izquierda a derecha.

ca, se corresponde con una muy buena aproximación con el resto de normas comparadas y con los modelos de dinámica de fluidos, en el momento en que se analizan edificios esbeltos y/o con frecuencias fundamentales en sentido transversal inferiores a $0,5 \mathrm{~Hz}$ es imprescindible llegar a cabo un análisis particularizado.

El estudio detecta un campo de frecuencias fundamentales donde el Eurocódigo no identifica un incremento significativo de la fuerza transversal que sin embargo si detectan las otras normas internacionales, sin llegar aún a producirse el acoplamiento de frecuencias, momento en que la fuerzas transversal se ve significativamente incrementada. Para los edificios de esbeltez igual o superior a 3 , y con frecuencias inferiores a $0,5 \mathrm{~Hz}$, existe un incremento progresivo en la acción eólica transversal a medida que el edificio se hace más flexible. Es a partir de un umbral de frecuencia mínimo, de- pendiente de la velocidad del flujo, la forma del edificio y su dimensión transversal al flujo, en que puede acontecer el acoplamiento de frecuencias, momento en el que las fuerzas eólicas transversales gobernaran cualquier aspecto del diseño de la estructura del edificio. Se ha visto, por ejemplo, que para edificios de $75 \mathrm{~m}$ de altura y 25x25m de dimensión en planta, la frecuencia fundamental es crítica para valores inferiores a $0,22 \mathrm{~Hz}$, mientras que para alturas de $150 \mathrm{~m}$ la frecuencia es crítica a partir de $0,24 \mathrm{~Hz}$.

Se considera fundamental el estudio e incorporación de las acciones eólicas transversales, analizadas mediante métodos que permitan su precisa consideración (Eurocódigo para el rango de edificios poco esbeltos o edificios en los que se produzca el acoplamiento de frecuencias, o bien haciendo uso de normas internacionales, estudios numéricos o túneles experimentales aeroelásticos).

\section{REFERENCIAS}

(1) Gu, M., Quan, Y. (2004). Across-wind loads of typical tall buildings. Journal of Wind Engineering and Industrial Aerodynamics, 92 (2004): 1147-1165.

(2) AENOR (2007). UNE-EN 1991-1-4:2007. Eurocódigo 1: Acciones en estructuras. Parte 1-4: Acciones generales. Acciones de viento.

(3) AS/NZS 1170.2:2011 (2011). Structural Design Actions. Part 2 - Wind Actions.

(4) AIJ Recomendations for Loads on Buildings (AIJ-RLB) (2004).

(5) Holmes J.D. (2014). Along- and Cross-wind response of a generic tall building: Comparison of wind-tunnel data with codes and standards. Journal of Wind Engineering and Industrial Aerodynamics 132 (2014): 136-141.

(6) Petersen, L., Mortensen, E., et al. (1998). Wind in the Atmospheric Boundary Layer. Part I - Climate and Turbulence (pp. 16-20). Wind Power Meteorology. RisØ National Laboratory. (RisØ-I-1206(EN).

(7) Rizk, A.S. (2010). Structural design of reinforced Concrete Tall Buildings. Council of Tall Buildings and Urban Habitat. CTBUH Journal, Issue I.

(8) Boggs, D., Dragovich, J. (2015). The Nature of Wind Loads and Dynamic Response (Chapter 2). SP.240: Performance Based Design of Concrete Buildings for Wind Loads. American Concrete Institute.

(9) Bachmann, H., Ammann, W., et. al. (1991) Vibrations problems in structures Bulletin no 209. Grupo V/4 Vibrations (Commission $V$-Serviceability). CEB (actual FIB). Documento original traducido por la Asociación Científico-Técnica del Hormigón Estructural: "Problemas de vibraciones en estructuras. (2001) Recomendaciones y manuals técnicos. Estructuras y Edificación (E-8)”.

(10) Tamura Y. (2013) Damping in Buildings and Estimation Techniques. Tamura Y., Kareem A. (eds) Advanced Structural Wind Engineering. Springer, Tokyo.

(11) Tamura Y. (2012). Amplitude Dependency of Damping in Buildings and Critical Tip Drift Ratio. International Journal of High-Rise Buildings. 1(1): 1-13. Council of Tall Buildings and Urban Habitat. CTBUH.

(12) Ruscheweyh, H., (1994) Vortex Excited Vibrations. Sockel, H. (Ed.), Wind-excited Vibrations of Structures (pp. 51-84). New York: Springer-Verlag, Wien. 
(13) Hansen, S. (2007, 2-7 de noviembre). Vortex-induced vibrations of structures. Structural Engineers World Congress. Bangalore. India.

(14) Castro, H.G., Bortoli, M.E., et al. (2015). Una metodología de cálculo para la determinación de la respuesta dinámica longitudinal de estructuras altas bajo la acción del viento. Revista internacional de Métodos Numéricos para Cálculo y diseño en Ingeniería. 31 (4): 235-245.

(15) Ruscheweyh, H. (1994). Vortex Excited Vibrations (pp. 51-84). Sockel, H. (Ed), Wind-Excited vibrations of Structures. International Centre for Mechanical Sciences. Courses and Lectures - No. 335. Springer-Verlag Wiem GMBH.

(16) Tamura, Y., et al. (2008). Peak normal stresses and effects of wind direction on wind load combinations for médium-rise buildings. Journal of Wind Engineering and Industrial Aerodynamics 96: 1043-1057.

(17) Proakis, J., Manolakis, G. (1998). Tratamiento Digital de Señales. Prentices-Hall Inc. 